\title{
Vacunas para COVID-19 - ¿Podemos evitar volver a vivir en un pueblo fantasma?
}

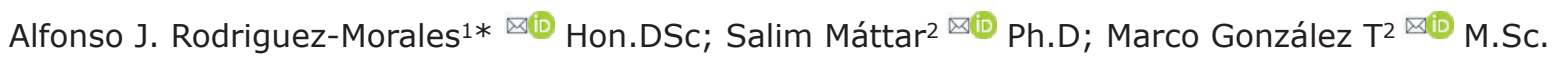

${ }^{1}$ Fundación Universitaria Autónoma de las Américas, Facultad de Medicina, Grupo de Investigación Biomedicina, Pereira, Risaralda, Colombia.

2Universidad de Córdoba, Facultad de Medicina Veterinaria y Zootecnia, Instituto de Investigaciones Biológicas del Trópico, Montería, Córdoba, Colombia.

*Correspondence: alfonso.rodriguez@uam.edu.co

Recibido: Marzo 2021; Aceptado: Marzo 2021; Publicado: Marzo 2021.

Durante el último año, la pandemia de la Enfermedad por Coronavirus 2019 (COVID-19), causada por el Síndrome Respiratorio Severo Coronavirus 2 (SARS-CoV-2), ha causado estragos considerables en la sociedad (1). De hecho, esta ha sido una crónica de una zoonosis anunciada $(2,3)$. Su impacto ha sido aún más profundo en países en desarrollo $(4,5)$, como Colombia $(6,7,8)$. Hasta el momento, hasta el 9 de marzo de 2021, el mundo ha registrado 117.5 millones de casos de COVID-19, con 2.6 millones de muertes (2.22\%). Colombia está incluida en el Top 20 de países con el mayor número de casos (2.282.372) y en el Top 20 de muertes a nivel mundial (60.676).

A pesar del número relativo de casos que evolucionan hacia desenlaces graves y fatales, dada la magnitud de la pandemia, su trascendencia clínica es tan relevante, ya que esta enfermedad emergente afecta de manera desproporcionada a personas mayores de 60 años y con factores de riesgo, especialmente en situación de pobreza. Entre ellos se concentran los casos graves y mortales.

Por todo ello, desde el inicio de la pandemia, hace más de un año, se comprendió la urgente necesidad de tratamientos y vacunas eficaces y efectivas $(9,10)$. Afortunadamente, en este camino de piedra, el rápido desarrollo de una gran cantidad de candidatos a vacunas COVID-19 allanó el camino para las esperanzas de control de la pandemia (11). Veintiuna vacunas llegaron a los ensayos de fase 3, seis de ellas autorizadas, de uso temprano o limitado, y seis aprobadas por agencias reguladoras internacionales, como la FDA y la EMA. Aún más, 30 están en camino en la fase 2 y 42 en la fase 1, sin mencionar docenas o cientos de candidatos preclínicos adicionales en estudio (11).

Entre ellas, las vacunas desarrolladas y producidas por Pfizer-Biontech, Gamaleya, Oxford-AstraZeneca, Sinopharm y Sinovac, se encuentran actualmente desplegadas en sus primeros lotes de dosis en América Latina, con la esperanza de alcanzar más temprano que tarde una cobertura poblacional significativa. Todas estas vacunas son significativamente eficaces (estudio hasta en ensayos de fase 3, controlados). Sin embargo, algunos de ellos han demostrado recientemente que son efectivos (en el mundo real), como lo es la vacuna Pfizer-Biontech BNT162b2 COVID-19. Esta vacuna, aplicada masivamente en Israel, demostró que es efectiva para una amplia gama de resultados relacionados con COVID-19, un hallazgo consistente con el de los ensayos aleatorizados $(12,13,14)$. Sin embargo, Colombia no es Israel. Y hasta ahora, nuestro plan de vacunación en este país sudamericano ha comenzado tarde y lento. 
En Colombia, hasta el 9 de marzo de 2021 se han aplicado poco más de 315.000 dosis de las vacunas Pfizer-Biontech y Sinovac COVID-19. El número de vacunas diarias no ha superado las 34.000 dosis diarias (Figura 1). A este ritmo, se necesitarán 1.530 días para vacunar a toda la población de Colombia ( 51 millones de personas), probablemente en abril de 2025 . Todos están preocupados y esperan aumentar el número de personas vacunadas diariamente en el país en al menos entre 100.000 y 150.000 . por día para alcanzar la meta en menos de un año. Eso representa un desafío complejo pero una prioridad importante para la nación y su salud pública.

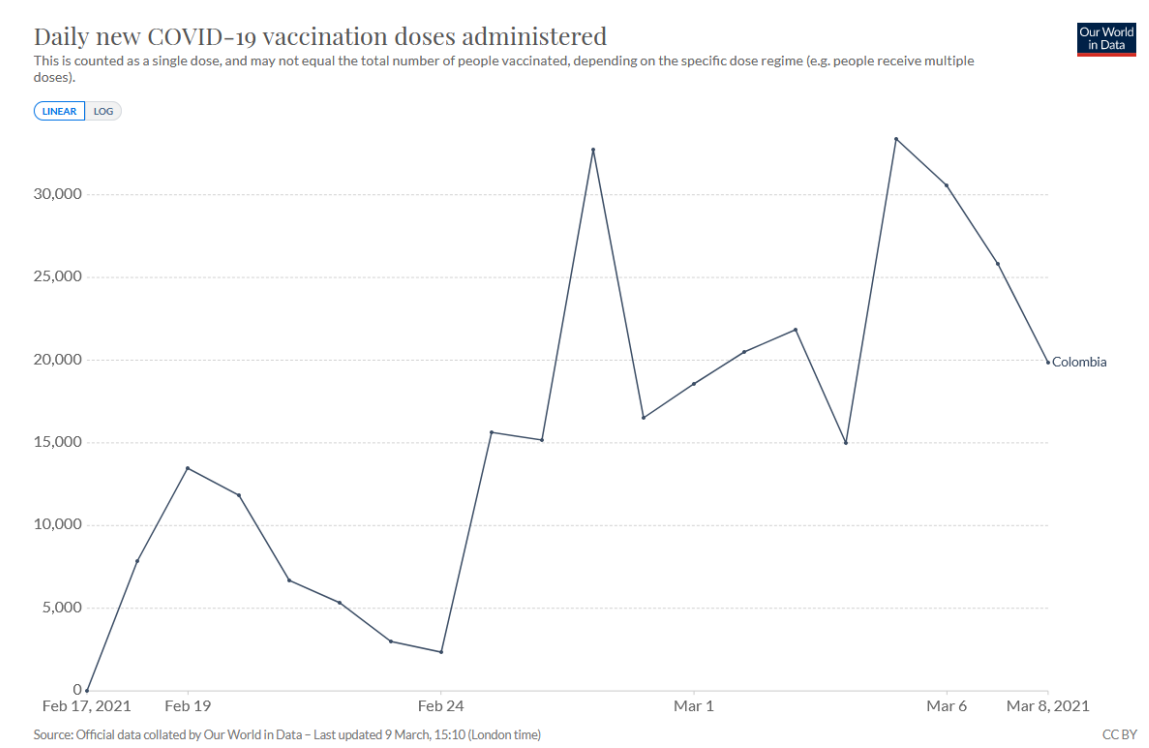

Figura 1. Nuevas dosis diarias de vacuna COVID-19 administradas en Colombia hasta el 8 de marzo de 2021.

Más allá de eso, múltiples expertos en la materia se preguntan si, con las vacunas COVID-19, ¿podemos evitar volver a vivir en un pueblo fantasma? En los peores momentos de esta pandemia, "todos nos encerramos", ya que la famosa banda británica de rock n'roll formada en Londres (Reino Unido) en 1962, Rolling Stones $\AA$, hizo referencia a la pandemia de COVID-19 y fue lanzada en abril 2020 en medio de las primeras oleadas de enfermedades. El año 2021 se considera un año de transición. Sin embargo, el control real de la pandemia se basa no solo en la eficacia y efectividad de las vacunas COVID-19 y los buenos planes nacionales de vacunación, sino también en mantener, bajo estricto cumplimiento las medidas de bioseguridad y afines, así como en educar y difundir correctamente la información relacionada a la relevancia y rol de las vacunas COVID-19 en este escenario. Muchas preguntas aún deben ser respondidas en detalle por la ciencia. Cuánto tiempo durará la inmunidad a la infección natural, a las vacunas, será necesario revacunar a las personas en un año, tal como ocurre con la influenza estacional. ¿Cómo se ven afectadas las vacunas por las nuevas variantes preocupantes del SARS-CoV-2, como la P.1, ya presente en Colombia, en Leticia, Amazonas? u otros que circulan en Reino Unido, Sudáfrica y muchos otros países? $(15,16)$. Nadie quiere volver a vivir en un "pueblo fantasma" en cuarentena, aislamiento o encierro, pero el futuro depende de nosotros. Esperamos que la ciencia vuelva a triunfar, como se hizo con muchas otras enfermedades epidémicas y pandémicas derrotadas por la humanidad en nuestra larga historia en la tierra, como la poliomielitis, el sarampión, la rubéola, la peste, entre muchas otras.

\section{REFERENCIAS}

1. Dhama K, Khan S, Tiwari R, Sircar S, Bhat S, Malik YS, et al. Coronavirus Disease 2019-COVID-19. Clin Microbiol Rev. 2020; 33(4):e00028-20. https://doi. org/10.1177/1470320320926902
2. Mattar V S, González Tous M. Emergencia zoonótica por coronavirus: riesgo potencial para la salud pública en America Latina. Rev MVZ Cordoba. 2018; 23(3):6775-6777. https://doi.org/10.21897/rmvz.1408 
3. Mattar S, González Tous M. Coronavirus: Crónica de una zoonosis anunciada. Rev MVZ Cordoba. 2020;25(2):e2048. https:// doi.org/10.21897/rmvz.2048

4. Rodriguez-Morales AJ, Gallego V, EscaleraAntezana JP, Mendez CA, Zambrano LI, Franco-Paredes C, et al. COVID-19 in Latin America: The implications of the first confirmed case in Brazil. Travel Med Infect Dis. 2020; 35:101613. https://doi. org/10.1016/j.tmaid.2020.101613

5. Cimerman S, Chebabo A, Cunha CAD, Rodriguez-Morales AJ. Deep impact of COVID-19 in the healthcare of Latin America: the case of Brazil. Braz J Infect Dis. 2020; 24(2):93-95. https://doi.org/10.1016/j. bjid.2020.04.005

6. Diaz-Guio DA, Villamil-Gomez WE, Dajud $L$, Perez-Diaz CE, Bonilla-Aldana DK, Mondragon-Cardona $A$, et al. Will the Colombian intensive care units collapse due to the COVID-19 pandemic? Travel Med Infect Dis. 2020; 38:101746. https://dx.doi. org/10.1016\%2Fj.tmaid.2020.101746

7. Mattar S, Martinez-Bravo C, Rivero R, Contreras H, Faccini-Martinez AA, GuzmanTeran $C$, et al. Epidemiological and viral features of a cohort of SARS-CoV-2 symptomatic and asymptomatic individuals in an area of the Colombian Caribbean. Ann Clin Microbiol Antimicrob. 2020; 19:58. https://doi.org/10.1186/s12941-020$\underline{00397-5}$

8. Mattar S, Alvis-Guzman N, Garay E, Rivero R, Garcia A, Botero Y, et al. Severe Acute Respiratory Syndrome Coronavirus 2 Seroprevalence Among Adults in a Tropical City of the Caribbean Area, Colombia: Are We Much Closer to Herd Immunity Than Developed Countries? Open Forum Infect Dis. 2020; 7(12): ofaa550. https://doi. org/10.1093/ofid/ofaa550

9. Cardona-Ospina JA, Rojas-Gallardo DM, Garzon-Castano SC, Jimenez-Posada EV, Rodriguez-Morales AJ. Phylodynamic analysis in the understanding of the current COVID-19 pandemic and its utility in vaccine and antiviral design and assessment. Hum Vaccin Immunother. 2021; 1-8. https://doi. org/10.1080/21645515.2021.1880254
10. Patel SK, Pathak M, Tiwari R, Yatoo MI, Malik YS, Sah $R$, et al. A vaccine is not too far for COVID-19. J Infect Dev Ctries. 2020; 14(5):450-453. https://doi.org/10.3855/ jidc. 12744

11. Rabaan AA, Al-Ahmed SH, Sah $\mathrm{R}, \mathrm{Al}$-Tawfiq JA, Al-Qaaneh AM, Al-Jamea LH, et al. Recent advances in vaccine and immunotherapy for COVID-19. Hum Vaccin Immunother. 2020; 16(12):3011-3022. https://doi.org/10.108 $\underline{0 / 21645515.2020 .1825896}$

12. Baden LR, El Sahly HM, Essink B, Kotloff K, Frey S, Novak R, et al. Efficacy and Safety of the mRNA-1273 SARS-CoV-2 Vaccine. N Engl J Med. 2021; 384(5):403-416. https:// doi.org/10.1056/NEJMoa2035389

13. Dagan N, Barda N, Kepten E, Miron O, Perchik S, Katz MA, et al. BNT162b2 mRNA Covid-19 Vaccine in a Nationwide Mass Vaccination Setting. N Engl J Med. 2021. https://doi.org/10.1056/NEJMoa2101765

14. Polack FP, Thomas SJ, Kitchin N, Absalon J, Gurtman A, Lockhart S, et al. Safety and Efficacy of the BNT162b2 mRNA Covid-19 Vaccine. N Engl J Med. 2020; 383(27):2603-15. https://doi.org/10.1056/ NEJMoa2034577

15. Fontanet $A$, Autran $B$, Lina $B$, Kieny MP, Karim SSA, Sridhar D. SARS-CoV-2 variants and ending the COVID-19 pandemic. Lancet. 2021; 397(10278):P952-954. https://doi. org/10.1016/S0140-6736(21)00370-6

16. Schlagenhauf $P$, Patel $D$, RodriguezMorales A, Gautret P, Grobusch MP, Leder K. Variants, Vaccines and Vaccination passports: Challenges and Chances for Travel Medicine in 2021. Travel Med Infect Dis. $2021 ; 40: 101996$. https://dx.doi. org/10.1016\%2Fj.tmaid.2021.101996 